\title{
THE UNIVERSAL ZETA FUNCTION FOR CURVE SINGULARITIES AND ITS RELATION WITH GLOBAL ZETA FUNCTIONS
}

\author{
JULIO JOSÉ MOYANO-FERNÁNDEZ
}

\begin{abstract}
The purpose of this note is to give a brief overview on zeta functions of curve singularities and to provide some evidences on how these and global zeta functions associated to singular algebraic curves over perfect fields relate to each other.
\end{abstract}

\section{INTRODUCTION}

1.1. Let $X$ be a complete, geometrically irreducible, singular algebraic curve defined over a perfect field $k$; from now on we will refer to such a curve simply as 'algebraic curve over $k$ '. Let $K$ be the field of rational functions on $X$. Extending previous works of V. M. Galkin and B. Green - and based on the classical results of F. K. Schmidt [23] for nonsingular curves - K.O. Stöhr (cf. [25], [26]) managed to attach a zeta function to $X$ for finite $k$ in the following manner: If $\mathcal{O}_{X}$ is the structure sheaf of $X$, he defined the Dirichlet series

$$
\zeta\left(\mathcal{O}_{X}, s\right):=\sum_{\mathfrak{a} \succeq \mathcal{O}_{X}} q^{-s \operatorname{deg} \mathfrak{a}}, \quad s \in \mathbb{C} \text { with } \operatorname{Re}(s)>0,
$$

where the sum is taken over all positive divisors of $X$, and $\operatorname{deg}(\cdot)$ denotes the degree of those divisors. Observe that the change of variables $T=q^{-s}$ allows to consider the formal power series in $T$

$$
Z\left(\mathcal{O}_{X}, T\right)=\sum_{n=0}^{\infty} \#(\{\text { positive divisors of } X \text { of degree } n\}) \cdot T^{n} .
$$

Moreover, Stöhr considered local zeta functions, i.e., zeta functions attached to every local ring $\mathcal{O}_{P}$ of points $P$ at $X$ of the form

$$
Z\left(\mathcal{O}_{P}, T\right):=\sum_{\mathfrak{a} \subseteq \mathcal{O}_{P}} T^{\operatorname{deg} \mathfrak{a}}=\sum_{n=0}^{\infty} \#\left(\left\{\text { positive } \mathcal{O}_{P} \text {-ideals of degree } n\right\}\right) \cdot T^{n} .
$$

This series extends previous definitions by Galkin [12] and Green [13]. Furthermore, the Euler product formula for the formal power series yields the identity

$$
Z\left(\mathcal{O}_{X}, T\right)=\prod_{P \in X} Z\left(\mathcal{O}_{P}, T\right)
$$

2010 Mathematics Subject Classification. Primary: 14H20, 14G10; Secondary: 32S40, 11S40. Key words and phrases. Zeta function, Curve singularity, Poincaré series, monodromy.

The author was partially supported by the Spanish Government Ministerio de Economía, Industria y Competitividad (MINECO), grants MTM2012-36917-C03-03 and MTM2015-65764C3-2-P, as well as by Universitat Jaume I, grant P1-1B2015-02. 
which actually establishes a link between the local and global theory. Every local factor $Z\left(\mathcal{O}_{P}, T\right)$ splits again into factors

$$
Z\left(\mathcal{O}_{P}, \mathcal{O}_{P}, T\right)=\sum_{n=0}^{\infty} \#(\{\text { principal integral } \mathcal{O} \text {-ideals of codimension } n\}) \cdot T^{n}
$$

which are determined by the value semigroup of $\mathcal{O}_{P}$ (see $\S 2.1$ for the definition of this semigroup) if the field is big enough, as Zúñiga showed in [29].

1.2. On the other hand, when studying the Gorenstein property of one-dimensional local Cohen-Macaulay rings, Campillo, Delgado and Kiyek [10, (3.8)] observed the existence of a Laurent series - a polynomial in their situation - attached to those rings, and satisfying a functional equation in the case of Gorenstein rings. Further investigations by Campillo, Delgado and Gusein-Zade [2]-[8] led to the definition of a Poincaré series associated to a complex curve singularity as an integral with respect to the Euler characteristic (se also O. Viro [28]). They even considered integration with respect to an Euler characteristic of motivic nature and so they introduced the notion of generalized Poincaré series of a complex curve singularity [9].

1.3. In the spirit of the preceding paragraphs, the author showed in his thesis [17] (see also the joint paper with his advisor Delgado [11]) that the factors $Z\left(\mathcal{O}_{P}, \mathcal{O}_{P}, T\right)$ coincide essentially with the generalized Poincare series of Campillo, Delgado and Gusein-Zade, under a suitable specialization for finite fields (see $§ 3.7$ below). These ideas have also provided some feedback: for instance Stöhr achieved a deeper insight into the nature of the local zeta functions (see [27], and [16] together with his student J.J. Mira).

1.4. The key ingredient that allows to relate those different formal power series is the universal zeta function for a curve singularity defined by Zúñiga and the author in [22]: for example, the local zeta functions and Poincaré series mentioned above are specializations of this universal zeta function. After some preliminaries, we devote Section 3 to describe this series. Moreover, we claim that one may establish the local-global behaviour explained in $\$ 1.1$ for curves defined over nonfinite fields. This conjectural behaviour has already shown some evidences in particular cases; see e.g. the theorem in Section 4.

\section{Preliminaries And NOtations}

2.1. Consider the normalization $\pi: \tilde{X} \rightarrow X$ of an algebraic curve $X$ over $k$, and let $\mathcal{O}=\mathcal{O}_{P}:=\mathcal{O}_{P, X}$ be the local ring of $X$ at $P$. For the sake of simplicity we will assume the ring $\mathcal{O}$ to be complete.

It is $\pi^{-1}(P)=\left\{Q_{1}, \ldots, Q_{d}\right\}$ and so the corresponding local rings $\mathcal{O}_{Q_{i}}$ are discrete valuation rings of $K$ over $\mathcal{O}$. The value semigroup associated to $\mathcal{O}$ is defined to be

$$
S(\mathcal{O}):=\{\underline{v}(\underline{z}): \underline{z} \text { nonzero divisor in } \mathcal{O}\} \subseteq \mathbb{N}^{d} ;
$$

here $\underline{v}(\underline{z})=\left(v_{1}\left(z_{1}\right), \ldots, v_{d}\left(z_{d}\right)\right)$, where each $v_{i}$ stands for the valuation associated with $\mathcal{O}_{Q_{i}}$; we write $S$ for this semigroup from now on. Let $c=c(S)$ denote the conductor of $S$, i.e. the smallest element $\underline{v} \in S$ such that $\underline{v}+\mathbb{N}^{d} \subseteq S$. Moreover, 
$\mathcal{O}^{\times}$denotes the group of units of $\mathcal{O}$. Further details here and in the sequel can be checked in [22] and the references therein.

2.2. We say that the ring $\mathcal{O}$ is totally rational if all $\operatorname{rings} \mathcal{O}_{Q_{i}}$, for $i=1, \ldots, d$ have $k$ as a residue field.

2.3. The integral closure of $\mathcal{O}$ in $K / k$ is $\tilde{\mathcal{O}}=\tilde{\mathcal{O}}_{P}=\mathcal{O}_{Q_{1}} \cap \ldots \cap \mathcal{O}_{Q_{d}}$. We write $\tilde{\mathcal{O}}^{\times}$for its group of units. The singularity degree $\delta_{P}$ of $\tilde{\mathcal{O}}$ is defined as $\delta_{P}=\delta:=\operatorname{dim}_{k} \tilde{\mathcal{O}} / \mathcal{O}<\infty$ (see e.g. [24, Chapter IV]).

2.4. For $\underline{n} \in S$ we set

$$
\mathcal{I}_{\underline{n}}:=\{I \subseteq \mathcal{O} \mid I=\underline{z} \mathcal{O}, \text { with } \underline{v}(\underline{z})=\underline{n}\},
$$

and for $m \in \mathbb{N}$,

$$
\mathcal{I}_{m}:=\bigcup_{\|\underline{n} \in \underline{n}\|=m} \mathcal{I}_{\underline{n}},
$$

where $\|\underline{n}\|$ denotes the sum of the components of the vector $\underline{n}=\left(n_{1}, \ldots, n_{d}\right) \in \mathbb{N}^{d}$.

2.5. In the category $\operatorname{Var}_{k}$ of $k$-algebraic varieties, we define the Grothendieck ring $K_{0}\left(\operatorname{Var}_{k}\right)$, which is the ring generated by symbols $[V]$ for $V \in \operatorname{Var}_{k}$, with the relations $[V]=[W]$ if $V$ is isomorphic to $W,[V]=[V \backslash Z]+[Z]$ if $Z$ is closed in $V$, and $[V \times W]=[V][W]$. We write $\mathbb{L}:=\left[\mathbb{A}_{k}^{1}\right]$ for the class of the affine line, and $\mathcal{M}_{k}:=K_{0}\left(\operatorname{Var}_{k}\right)\left[\mathbb{L}^{-1}\right]$ for the ring obtained by localization with respect to the multiplicative set generated by $\mathbb{L}$.

2.6. It is possible to associate to $\mathcal{I}_{\underline{\underline{n}}}$ resp. $\mathcal{I}_{m}$ well-defined classes in the Grothendieck ring [22, Section 5]; those classes will be denoted by $\left[\mathcal{I}_{\underline{n}}\right]$ resp. $\left[\mathcal{I}_{m}\right]$. This allows to attach to the local ring $\mathcal{O}$ the zeta functions

$$
Z\left(T_{1}, \ldots, T_{d}, \mathcal{O}\right):=\sum_{\underline{n} \in S}\left[\mathcal{I}_{\underline{n}}\right] \mathbb{L}^{-\|\underline{n}\|} T^{\underline{n}} \in \mathcal{M}_{k} \llbracket T_{1}, \ldots, T_{d} \rrbracket,
$$

where $T^{\underline{n}}:=T_{1}^{n_{1}} \cdot \ldots \cdot T_{d}^{n_{d}}$, and $Z(T, \mathcal{O}):=Z(T, \ldots, T, \mathcal{O})$.

2.7. Definition. Consider an algebraic curve $X$ over $k$. If $k$ has characteristic $p \geq 0$, then we say that $k$ is big enough for $X$ if for every singular point $P$ in $X$ the following two conditions hold: 1$)$ the $\operatorname{ring} \mathcal{O}$ is totally rational and 2) $\widetilde{\mathcal{O}}^{\times} / \mathcal{O}^{\times} \cong\left(G_{m}\right)^{d-1} \times\left(G_{a}\right)^{\delta-d+1}$, with $G_{m}=\left(k^{\times}, \cdot\right)$ and $G_{a}=(k,+)$.

Note that the condition ' $k$ is big enough for $X$ ' is fulfilled when $p$ is big enough.

\section{The UnIVERSAL ZETA FUNCTION FOR CURVE SINGULARITIES}

3.1. For $k=\mathbb{C}$, we consider a semigroup $S \subseteq \mathbb{N}^{d}$ such that $S=S(\mathcal{O})$. Moreover, for $\underline{n} \in S$ set

$$
\mathcal{I}_{\underline{\underline{n}}}(U):=(U-1)^{-1} U^{\|\underline{n}\|+1} \sum_{I \subseteq[d]}(-1)^{\#(I)} U^{-\operatorname{dim}_{k}\left(\mathcal{O} /\left\{\underline{z} \in \mathcal{O}: \underline{v}(\underline{z}) \geqslant \underline{n}+\underline{1}_{I}\right\}\right)},
$$

for an indeterminate $U$, and where $[d]:=\{1,2, \ldots, d\}$, and $\underline{1}_{I}$ is the $d$-tuple with the components corresponding to the indices in $I$ equal to 1 , and the other components equal to 0 .

The notation $\mathcal{I}_{\underline{n}}(U)$ is appropriate, since that expression coincides with $\left[\mathcal{I}_{\underline{n}}\right]$ when $U$ specializes to $\mathbb{L}$, cf. [22, Section 5]. 
3.2. Let $\underline{c}=\left(c_{1}, \ldots, c_{d}\right)$ be the conductor of the semigroup $S$, cf. $\S 2.1$. Let $J:=\{1, \ldots, r\} \subseteq[d]$, and let $\underline{m} \in \mathbb{N}^{d}$ be such that $\underline{c}>\underline{m}$, i.e., $c_{i}>m_{i}$ for all $i \in[d]$. For a fixed $\emptyset \subsetneq J \subsetneq[d]$, set $r_{J}:=\# J$ and

$$
B_{J}:=\left\{\underline{m} \in \mathbb{N}^{r_{J}}: H_{J, \underline{m}} \neq \emptyset\right\},
$$

where $H_{J, \underline{m}}:=\left\{\underline{n} \in S: n_{j} \geq c_{j}\right.$ if $j \in J$, and $n_{j}=m_{j}$ otherwise $\}$.

Definition We define the universal zeta function $\mathcal{Z}\left(T_{1}, \ldots, T_{d}, U, S\right)$ associated with $S$ to be

$$
\begin{aligned}
\sum_{\underline{\underline{n} \in S} \leqslant \underline{n}<\underline{c}} \mathcal{I}_{\underline{n}}(U) U^{-\|\underline{n}\|} T^{\underline{n}}+\sum_{\emptyset \subseteq J \subsetneq I_{0}} \sum_{\underline{m} \in B_{J}}(U-1) U^{\|\underline{c}\|-\delta-1} \mathcal{I}_{\underline{f}_{J}(\underline{m})}(U) U^{-\|\underline{c}\|-\left\|\underline{f_{J}} \underline{(\underline{m})}\right\|_{\times}} \\
\times \frac{T \underline{f_{J}(\underline{m})}}{\prod_{i=1}^{r_{J}}\left(1-U^{-1} T_{i}\right)}+\frac{(U-1)^{d-1} U^{\delta-d+1} U^{-\|\underline{c}\|} T^{\underline{c}}}{\prod_{i=1}^{d}\left(1-U^{-1} T_{i}\right)}
\end{aligned}
$$

where $f_{J}(\underline{m})=\left(c_{1}, \ldots, c_{r_{J}}, m_{r_{J}+1}, \ldots, m_{d}\right) \in S$, with $m_{i}<c_{i}, r_{J}+1 \leqslant i \leq d$, and $1 \leqslant r_{J}<d$.

3.3. Observe that this universal zeta function is completely determined by $S$. The adjective universal applied to this zeta function will be clear after the following paragraphs.

3.4. The generalized Poincaré series $P_{g}\left(T_{1}, \ldots, T_{d}\right)$ of Campillo, Delgado and Gusein-Zade ([9]; see also [10], [11]) as an integral with respect to an Euler characteristic of motivic nature is very close to the zeta function $Z\left(T_{1}, \ldots, T_{d}, \mathcal{O}\right)$ of $\S 2.6$, and therefore to the universal zeta function via the specialization $U=\mathbb{L}$ :

Proposition. If $S=S(\mathcal{O})$ and $k$ is big enough for $Y$, then

$$
Z\left(T_{1}, \ldots, T_{d}, \mathcal{O}\right)=\mathbb{L}^{\delta+1} P_{g}\left(T_{1}, \ldots, T_{d}\right)=\left.\mathcal{Z}\left(T_{1}, \ldots, T_{d}, U, S\right)\right|_{U=\mathbb{L}} .
$$

3.5. In addition, a certain specialization of the universal zeta function coincides with the zeta function of the monodromy transformation of a reduced plane curve singularity acting on its Milnor fibre, as we briefly explain now.

Definition. Let $(X, 0) \subseteq\left(\mathbb{C}^{2}, 0\right)$ be a reduced plane curve singularity defined by an equation $f=0$, with $f \in \mathcal{O}_{\left(\mathbb{C}^{2}, 0\right)}$ reduced. Let $h_{f}: V_{f} \rightarrow V_{f}$ be the monodromy transformation of the singularity $f$ acting on its Milnor fiber $V_{f}$. The zeta function of the monodromy $h_{f}$ is defined to be

$$
\varsigma_{f}(T):=\prod_{i \geqslant 0}\left[\operatorname{det}\left(\mathrm{id}-\left.T \cdot\left(h_{f}\right)_{*}\right|_{H_{i}\left(V_{f} ; \mathbb{C}\right)}\right)\right]^{(-1)^{i+1}} .
$$

A result of Campillo, Delgado and Gusein-Zade ([2, Theorem 1]) allows us to prove:

Theorem. Let $k=\mathbb{C}$. Then for every $\mathcal{O}=\mathcal{O}_{\left(\mathbb{C}^{2}, 0\right)} /(f)$, with $f \in \mathcal{O}_{\left(\mathbb{C}^{2}, 0\right)}$ reduced, and for every $S=S(\mathcal{O})$, we have

$$
\begin{aligned}
& \varsigma_{f}(T)=\mathcal{Z}\left(T_{1}, \ldots, T_{d}, U, S\right) \mid T_{1}=\ldots=T_{d}=T \\
& U=1
\end{aligned}
$$


3.6. In [29] Zúniga introduced a Dirichlet series $Z(\mathrm{Ca}(X), T)$ associated to the effective Cartier divisors on an algebraic curve $X$ defined over $k=\mathbb{F}_{q}$, which admits an Euler product of the form

$$
Z(\mathrm{Ca}(X), T)=\prod_{P \in X} Z_{\mathrm{Ca}(X)}\left(T, q, \mathcal{O}_{P, X}\right),
$$

with $Z_{\mathrm{Ca}(X)}\left(T, q, \mathcal{O}_{P, X}\right):=\sum_{I \subseteq \mathcal{O}_{P, X}} T^{\operatorname{dim}_{k}\left(\mathcal{O}_{P, X} / I\right)}$, where $I$ runs through all the principal ideals of $\mathcal{O}_{P, X}$. In addition, $Z_{\mathrm{Ca}(X)}\left(T, q, \mathcal{O}_{P, X}\right)=Z\left(T, \mathcal{O}_{P, X}\right)$, cf. §2.6.

Observe that this zeta function is nothing but the zeta function $Z(\mathcal{O}, \mathcal{O}, T)$ appearing as a local factor in the Stöhr zeta function, cf. Section 1.

3.7. Remark. In the category of $\mathbb{F}_{q}$-algebraic varieties, $[\cdot]$ specializes to the counting rational points additive invariant $\#(\cdot)$. We write $\#\left(Z\left(T_{1}, \ldots, T_{d}, \mathcal{O}\right)\right)$ for the rational function obtained by specializing [.] to \# $(\cdot)$. From a computational point of view, $\#\left(Z\left(T_{1}, \ldots, T_{d}, \mathcal{O}\right)\right)$ is obtained from $Z\left(T_{1}, \ldots, T_{d}, \mathcal{O}\right)$ by replacing $\mathbb{L}$ by $q$.

3.8. Theorem. Let $k=\mathbb{F}_{q}$ and let $\mathcal{Z}\left(T_{1}, \ldots, T_{d}, U, S\right)$ be the universal zeta function for $S$. Moreover, let $X$ be an algebraic curve defined over $k$, and let $\mathcal{O}_{P, X}$ be the (complete) local ring of $X$ at a singular point $P$. Assume that $k$ is big enough for $X$ and that $S=S\left(\mathcal{O}_{P, X}\right)$.

(1) For any $\mathcal{O}=\mathcal{O}_{\left(\mathbb{C}^{2}, 0\right)} /(f)$, with $f \in \mathcal{O}_{\left(\mathbb{C}^{2}, 0\right)}$ reduced, and $S=S(\mathcal{O})$,

$$
\begin{gathered}
Z_{\mathrm{Ca}(X)}\left(q^{-1} T, q, \mathcal{O}_{P, X}\right)=\#\left(Z\left(T_{1}, \ldots, T_{d}, \mathcal{O}_{P, X}\right)\right) \\
=\mathcal{Z}\left(T_{1}, \ldots, T_{d}, U, S\right) \mid \begin{array}{l} 
\\
T_{1}=\ldots=T_{d}=T \\
U=q
\end{array}
\end{gathered}
$$

In particular $Z_{\mathrm{Ca}(X)}\left(q^{-1} T, q, \mathcal{O}_{P, X}\right)$ depends only on $S$. Moreover, if $X$ is plane, then $Z_{\mathrm{Ca}(X)}\left(q^{-1} T, q, \mathcal{O}_{P, X}\right)$ is a complete invariant of the equisingularity class of $\mathcal{O}_{P, X}$.

(2) For any $\mathcal{O}=\mathcal{O}_{\left(\mathbb{C}^{2}, 0\right)} /(f)$, with $f \in \mathcal{O}_{\left(\mathbb{C}^{2}, 0\right)}$, it holds that

$$
\left.Z_{\mathrm{Ca}(X)}\left(q^{-1} T, q, \mathcal{O}_{P, X}\right)\right|_{q \rightarrow 1}=\varsigma_{f}(T) .
$$

\section{Some Connections Between local And GLOBAl Zeta FunCtions}

4.1. For a smooth algebraic variety $Y$ defined over a field $k, \mathrm{M}$. Kapranov defined a zeta function as the formal power series in an indeterminate $u$

$$
\zeta_{\mathrm{mot}, Y}(u)=\sum_{n=0}^{\infty}\left[Y^{(n)}\right] u^{n} \in K_{0}\left(\operatorname{Var}_{k}\right) \llbracket u \rrbracket,
$$

where $Y^{(n)}$ stands for the $n$-fold symmetric product of $Y$ (cf. [14, §1]). (For instance, if $k=\mathbb{F}_{q}$, then one obtains the usual Hasse-Weil zeta function of $Y$, 
cf. §3.7). When $Y$ is a curve, Baldassarri, Deninger and Naumann introduced in [1] a two-variable version of the Kapranov zeta function, namely

$$
Z_{\mathrm{mot}, Y}(t, u)=\sum_{n, \nu \geqslant 0}\left[\mathrm{Pic}_{\nu}^{n}\right] \frac{u^{\nu}-1}{u-1} t^{n} \in K_{0}\left(\operatorname{Var}_{k}\right) \llbracket u, t \rrbracket,
$$

where the algebraic $k$-scheme $\operatorname{Pic}_{\nu}^{n}=\operatorname{Pic}_{\geqslant \nu}^{n} \backslash \operatorname{Pic}_{\geqslant \nu+1}^{n}$ (with $\operatorname{Pic}_{\geqslant \nu}^{n}$ being the closed subvariety - in the Picard variety of degree $n$ line bundles on $Y$ - of line bundles $\mathcal{L}$ with $\left.h^{0}(\mathcal{L}) \geqslant \nu\right)$ defines a class in $K_{0}\left(\operatorname{Var}_{k}\right)$.

4.2. The connections between the universal zeta function and the motivic zeta functions of Kapranov and Baldassarri-Deninger-Naumann are being currently investigated by A. Melle, W. Zúniga and the author; we believe that the zeta functions discussed in the previous sections are factors of motivic zeta functions of Baldassarri-Deninger-Naumann type for singular curves (as mentioned before, this is known when $k=\mathbb{F}_{q}$ ). In order to give some evidence supporting this belief, this note will be finished by stating the relation between local and global zeta functions in a particular situation.

The context will be the one of a modulus: Following Serre [24], let $k$ be an algebraically closed field, and let $C$ be an irreducible, non-singular, complete algebraic curve defined over $k$. If $F$ is a finite subset of $C$, a modulus $\mathfrak{m}$ supported on $F$ is defined to be the assignment of an integer $n_{P}>0$ for each point $P \in F$; this is sometimes identified with the effective divisor $\sum_{P} n_{P} P$.

4.3. It is possible to attach a curve to $\mathfrak{m}$ starting from $C$, essentially by "placing" the points in $F$ all together into one (see again [24]). The resulting singular curve $C_{\mathfrak{m}}$ has then this point as its only singularity. It holds the following

Theorem. Let $C_{\mathfrak{m}}$ be a curve arising from a modulus $\mathfrak{m}$ supported on a finite set of points of a curve $C$ as above, and let $P$ be its only singular point. Furthermore, let $\pi: \widetilde{C_{\mathfrak{m}}} \rightarrow C_{\mathfrak{m}}$ be the normalization morphism. Then

$$
Z_{\mathrm{mot}, C_{\mathfrak{m}}}\left(\mathbb{L}^{-1} T, \mathbb{L}\right)=Z_{\mathrm{mot}, \widetilde{C_{\mathfrak{m}}}}\left(\mathbb{L}^{-1} T, \mathbb{L}\right) \prod_{i=1}^{\sharp\left(\pi^{-1}(P)\right)}\left(1-\mathbb{L}^{-1} T\right) \cdot Z\left(T, \mathcal{O}_{P}\right) .
$$

The proof of this statement will appear in a forthcoming paper.

\section{REFERENCES}

[1] F. Baldassarri, C. Deninger, N. Naumann, "A motivic version of Pellikaan's two variable zeta function", in Diophantine Geometry, CRM Series 4, Ed. Norm., Pisa, 2007, 35-43.

[2] A. Campillo, F. Delgado, S. Gusein-Zade, On the monodromy of a plane curve singularity and the Poincaré series of its ring of functions. (Russian) Funktsional. Anal. i Prilozhen. 33 (1999), no. 1, 66-68; translation in Funct. Anal. Appl. 33 (1999), no. 1, 56-57.

[3] A. Campillo, F. Delgado, S. Gusein-Zade, The Alexander polynomial of a plane curve singularity, and the ring of functions on the curve. (Russian) Uspekhi Mat. Nauk 54 (1999), no. 3(327), 157-158; translation in Russian Math. Surveys 54 (1999), no. 3, 634-635.

[4] A. Campillo, F. Delgado, S. Gusein-Zade, Integrals with respect to the Euler characteristic over spaces of functions, and the Alexander polynomial. (Russian) Tr. Mat. Inst. Steklova 238 (2002), Monodromiya v Zadachakh Algebr. Geom. i Differ. Uravn., 144-157; translation in Proc. Steklov Inst. Math. 2002, no. 3 (238), 134-147. 
[5] A. Campillo, F. Delgado, S. Gusein-Zade, The Alexander polynomial of a plane curve singularity and integrals with respect to the Euler characteristic. Internat. J. Math. 14 (2003), no. 1, 47-54.

[6] A. Campillo, F. Delgado, S. Gusein-Zade, The Alexander polynomial of a plane curve singularity via the ring of functions on it. Duke Math. J. 117 (2003), no. 1, 125-156.

[7] A. Campillo, F. Delgado, S. Gusein-Zade, On the zeta functions of a meromorphic germ in two variables. Geometry, topology, and mathematical physics, 67-74, Amer. Math. Soc. Transl. Ser. 2, 212, Amer. Math. Soc., Providence, RI, 2004.

[8] A. Campillo, F. Delgado, S. Gusein-Zade, Poincaré series of curves on rational surface singularities. Comment. Math. Helv. 80 (2005), no. 1, 95-102.

[9] A. Campillo, F. Delgado, S. Gusein-Zade, Multi-index filtrations and generalized Poincaré series. Monatsh. Math. 150 (2007), no. 3, 193-209.

[10] A. Campillo, F. Delgado, K. Kiyek, Gorenstein property and symmetry for one-dimensional local Cohen-Macaulay rings. Manuscripta Math. 83 (1994), no. 3-4, 405-423.

[11] F. Delgado, J.J. Moyano-Fernández, On the relation between the generalized Poincaré series and the Stöhr zeta function. Proc. Amer. Math. Soc. 137 (2009), no. 1, 51-59.

[12] V. M. Galkin, Zeta functions of some one-dimensional rings, Izv. Akad. Nauk. SSSR Ser. Mat. 37 (1973), 3-19.

[13] B. Green, Functional equations for zeta-functions of non-Gorenstein orders in global fields, Manuscripta Math. 64 (1989), 485-502.

[14] M. Kapranov, The elliptic curve in the $S$-duality theory and Eisenstein series for KacMoody groups, preprint, arXiv:math/0001005 [math.AG].

[15] K. Kiyek, J.J. Moyano-Fernández, The Poincaré series of a simple complete ideal of a two-dimensional regular local ring. J. Pure Appl. Algebra 213 (2009), no. 9, 1777-1787.

[16] J.J. Mira, K.O. Stöhr, On Poincaré series of singularities of algebraic curves over finite fields. Manuscripta Math. 147 (2015), no. 3-4, 527-546.

[17] J.J. Moyano-Fernández, Poincaré series associated with curves defined over perfect fields. PhD Thesis, Universidad de Valladolid, 2008.

[18] J.J. Moyano-Fernández, Curvettes and clusters of infinitely near points. Rev. Mat. Complut. 24 (2011), no. 2, 439-463.

[19] J.J. Moyano-Fernández, Generalised Poincaré series and embedded resolution of curves. Monatsh. Math. 164 (2011), no. 2, 201-224.

[20] J.J. Moyano-Fernández, Poincaré series for curve singularities and its behaviour under projections. J. Pure Appl. Algebra 219 (2015), no. 6, 2449-2462.

[21] J.J. Moyano-Fernández, Fractional ideals and integration with respect to the generalised Euler characteristic. Monatsh. Math. 176 (2015), no. 3, 459-479.

[22] J.J. Moyano-Fernández, W.A. Zúñiga-Galindo, Motivic zeta functions for curve singularities. Nagoya Math. J. 198 (2010), 47-75.

[23] F. K. Schmidt, Analytische Zahlentheorie in Körpern der Charakteristik p, Math. Z. 33 (1931), 1-32.

[24] J.-P. Serre, Algebraic groups and class fields. Graduate Texts in Mathematics, 117. Springer, New York-Berlin-Heidelberg, 1988.

[25] K.O. Stöhr, On the poles of regular differentials of singular curves, Bol. Soc. Brasil. Mat. (N.S.) 24 (1993), no. 1, 105-136

[26] K.O. Stöhr, Local and global zeta-functions of singular algebraic curves, J. Number Theory 71 (1998), no. 2, 172-202.

[27] K.O. Stöhr, Multi-variable Poincaré series of algebraic curve singularities over finite fields. Math. Z. 262 (2009), no. 4, 849-866.

[28] O. Viro, Some integral calculus based on Euler characteristic. In: O. Ya. Viro (ed.), Topology and Geometry-Rohlin Seminar. Lecture Notes in Math. 1346, Springer, BerlinHeidelberg, 1988.

[29] W.A. Zúñiga-Galindo, Zeta functions and Cartier divisors on singular curves over finite fields, Manuscripta Math. 94 (1997), 75-88.

Universitat Jaume I, Campus de Riu Sec, Departamento de Matemáticas \& Institut Universitari de Matemàtiques i Aplicacions de Castelló, 12071 Castellón DE la Plana, Spain

E-mail address: moyano@uji.es 